\title{
Sulfone Containing Covalent Organic Frameworks for Photocatalytic Hydrogen Evolution from Water
}

\author{
Xiaoyan Wang ${ }^{1}$, Linjiang Chen ${ }^{1,2}$, Samantha Y. Chong ${ }^{1}$, Marc A. Little ${ }^{1}$, Yongzhen Wu ${ }^{3}$, \\ Wei-Hong Zhu ${ }^{3}$, Rob Clowes ${ }^{1}$, Yong Yan ${ }^{1}$, Martijn A. Zwijnenburg ${ }^{4}$, Reiner Sebastian \\ Sprick $^{1}$, and Andrew I. Cooper ${ }^{1,2, *}$ \\ ${ }^{1}$ Department of Chemistry and Materials Innovation Factory, University of Liverpool, \\ 51 Oxford Street, Liverpool, L7 3NY, UK \\ ${ }^{2}$ Leverhulme Research Centre for Functional Materials Design, Materials Innovation Factory \\ and Department of Chemistry, University of Liverpool, 51 Oxford Street, Liverpool, L7 3NY, \\ UK \\ ${ }^{3}$ Key Laboratory for Advanced Materials and Institute of Fine Chemicals, East China \\ University of Science and Technology, Meilong Road 130, Shanghai 200237, P. R. China \\ ${ }^{4}$ Department of Chemistry, University College London, 20 Gordon Street, London, WC1H 0AJ, \\ UK
}

\begin{abstract}
Nature uses organic molecules for light harvesting and photosynthesis but most man-made water splitting catalysts are inorganic semiconductors. Organic photocatalysts, while attractive because of their synthetic tunability, tend to have low quantum efficiencies for water splitting. Here we present a crystalline covalent organic framework (COF) based on a benzobis(benzothiophene sulfone) moiety that shows a much higher activity for photochemical hydrogen evolution than its amorphous or semi-crystalline counterparts. The COF is stable under long-term visible irradiation and shows steady photochemical hydrogen evolution with a sacrificial electron donor for at least fifty hours. We attribute its high quantum efficiency of FSCOF to its crystallinity, its strong visible light absorption, and its wettable, hydrophilic $3.2 \mathrm{~nm}$ mesopores. These pores allow the framework to be dye sensitized, leading to a further $61 \%$ enhancement in the hydrogen evolution rate up to $16.3 \mathrm{mmol} \mathrm{g}^{-1} \mathrm{~h}^{-1}$. The COF also retained its photocatalytic activity when cast as a thin film onto a support.
\end{abstract}


Photocatalytic solar hydrogen production—or water splitting —offers an abundant clean energy source for the future. The use of dispersed, powdered photocatalysts or thin catalyst films is attractively simple, but so far, no catalyst satisfies the combined requirements of cost, stability and solar-to-hydrogen efficiency. Since the first report of $\mathrm{TiO}_{2}$ as a photocatalyst, ${ }^{1}$ many inorganic semiconductors have been explored for water splitting, both in photoelectrochemical cells and as photocatalyst suspensions..$^{2-4}$ Recently, organic semiconductors have emerged as promising materials for photocatalytic hydrogen and oxygen evolution. ${ }^{5-7}$ Poly $(p$-phenylene) was first reported as a photocatalyst for hydrogen evolution in $1985,{ }^{8,9}$ but its activity was poor and limited to the ultraviolet spectrum. Since then, more active organic materials have been reported as visible light photocatalysts for hydrogen production using sacrificial donors. This started with carbon nitrides ${ }^{5,10}$ followed by poly(azomethine)s, ${ }^{11}$ conjugated microporous polymers (CMPs), ${ }^{6,12,13}$ linear conjugated polymers, ${ }^{12,14-16}$ and covalent triazine-based frameworks (CTFs). ${ }^{17-19}$ Carbon nitrides were further developed into hybrid systems that facilitate overall water splitting to produce both hydrogen and oxygen, for example by including metal co-catalysts. ${ }^{20} \mathrm{CMPs}$ were also claimed to exhibit overall photocatalytic water splitting. ${ }^{21}$ However, while it is possible to tune semiconductor properties such as band gap by modular copolymerization strategies ${ }^{6}$ organic materials such as carbon nitrides, conjugated polymers and CTFs lack long-range order: they are amorphous or semi-crystalline. ${ }^{17,22}$ This lack of order might limit the transport of photoactive charges to the catalyst surface. ${ }^{23}$ More generally, it is challenging to construct atomistic structure-property relationships for materials where the three-dimensional architecture is poorly defined.

Covalent organic frameworks $(\mathrm{COFs})^{24-26}$ are a class of organic materials that combine crystallinity, modular synthetic versatility, high accessible surface areas, and, sometimes, good physicochemical stability. ${ }^{27-30}$ With suitable building blocks and layered stacking sequences, COFs have been shown to have high charge-carrier mobilities. ${ }^{31}$ So far, only a small number of COFs have been studied for their photocatalytic hydrogen evolution activity. ${ }^{32,33}$ A hydrazonebased COF was reported by Lotsch and co-workers in 2014 to be active for sacrificial hydrogen production $^{34}$ and this team subsequently reported a series of 2D azine COFs with impressive photocatalytic hydrogen evolution rates of up to $1.7 \mathrm{mmol} \mathrm{g}^{-1} \mathrm{~h}^{-1}\left(\mathbf{N}_{3}\right.$-COF; Fig. 1a) using 
triethanolamine as a sacrificial donor and a platinum cocatalyst. ${ }^{35,36}$ Recently, Thomas and coworkers described a diacetylene functionalized COF with good hydrogen evolution activity. ${ }^{37}$ Kurongot and Banerjee reported a cadmium sulfide-COF composite material with a higher hydrogen evolution rate of $3.68 \mathrm{mmol} \mathrm{g}^{-1} \mathrm{~h}^{-1}$ using lactic acid as the sacrificial agent, ${ }^{32}$ although the COF was the minor component in that composite ( $90 \mathrm{wt}$ \% CdS). Porous COFs can also be modified after synthesis: for example, a molecular cobaloxime co-catalyst was introduced into an azine COF to give a hydrogen evolution rate of $0.78 \mathrm{mmol} \mathrm{g}^{-1} \mathrm{~h}^{-1} \cdot{ }^{38}$ Hydrogen evolution rates for a given catalyst can vary substantially in different laboratories depending on the optical set up; with that caveat in mind, $\mathbf{N}_{3}-\mathbf{C O F}$ is the most active, unmodified COF for sacrificial hydrogen evolution reported so far. ${ }^{35} \mathrm{COF}$ films have also been used as carbon dioxide reduction catalysts ${ }^{39}$ and, in film form, as photoelectrodes for light-driven water splitting. ${ }^{40}$

We showed before that a linear conjugated copolymer, P7 (Fig. 1b), exhibits hydrogen evolution rates of up to $1.49 \mathrm{mmol} \mathrm{g}^{-1} \mathrm{~h}^{-1}$ with a sacrificial amine donor under visible irradiation $(\lambda>420 \mathrm{~nm}) ;{ }^{16}$ that is, close to the value reported for $\mathbf{N}_{\mathbf{3}} \mathbf{- C O F} .{ }^{35}$ We ascribed this to the rigid, planar dibenzo $[b, d]$ thiophene sulfone (DBTS) unit in the $\mathbf{P} 7$ copolymer. Homopolymerization of this DBTS monomer gives a polymer, P10 (Fig. 1b), with an even higher hydrogen evolution rate (3.26 mmol g ${ }^{-1} \mathrm{~h}^{-1}$, Supplementary Fig. 95). Unlike COFs, these polymers are semicrystalline: they are also non-porous and insoluble, which precludes post-synthetic modification strategies. Here, we set out to incorporate the DBTS unit into ordered COFs to investigate the influence of crystallinity and porosity on the photocatalytic activity. This led to a new crystalline organic material, FS-COF, which is a better photocatalyst than equivalent amorphous or semi-crystalline conjugated polymers. FS-COF also exhibits higher hydrogen evolution rates than other COFs studied so far; up to $16.3 \mathrm{mmol} \mathrm{g}^{-1} \mathrm{~h}^{-1}$ when dye-sensitized, which is almost ten times higher than $\mathbf{N}_{3}-\mathbf{C O F}$. 


\section{RESULTS AND DISCUSSION}

\section{COF synthesis and characterization}

Three COFs, S-COF, FS-COF and TP-COF (Fig. 1c), were synthesized via a Schiff-base condensation reaction of 1,3,5-triformylphloroglucinol with aromatic diamines (Supplementary Figs 1-3). The products undergo an irreversible keto-enol tautomerization, which enhances their chemical stability. ${ }^{29}$ We used 3,7-diaminodibenzo[b,d]thiophene sulfone (SA) as the monomer for $\mathbf{S - C O F}$, which is a crystalline COF analogue of our semi-crystalline polymers $\mathbf{P 7}$ and P10 (Fig. 1b). We also used 3,9-diamino-benzo[1,2-b:4,5-b']bis[1]benzothiophene sulfone (FSA) to produce FS-COF, which has fused, extended planar linkers. TP-COF was synthesized as before; ${ }^{41}$ it was prepared from $4,4^{\prime \prime}$-diamino-p-terphenyl (TPA) and it is, in essence, FS-COF minus the sulfone moieities (Fig. 1c).

All three linkers gave rise to crystalline COFs (Fig. 2, Supplementary Figs 24-28). Based on PXRD data, FS-COF (Fig. 2c) appeared to have more long-range order than either S-COF (Fig. 2d) or TP-COF (Supplementary Fig. 28). This might be because the $\mathrm{C}-\mathrm{N}$ bond in the FSA monomer are parallel whereas the angle between the $\mathrm{C}-\mathrm{N}$ bonds in the $\mathbf{S A}$ monomer is $\sim 163^{\circ}$ (Supplementary Fig. 14), and hence the regular hexagonal framework in FS-COF may be less sensitive than S-COF to the insertion of linkers in the 'wrong' geometry. More effective $\pi-\pi$ stacking between the fused, planar FSA linkers might also help to stabilize FS-COF.

Powder X-ray diffraction (PXRD) was used to characterize the three COFs. FS-COF exhibited diffraction peaks at $2.71^{\circ}, 4.73^{\circ}, 5.52^{\circ}, 7.35^{\circ}$, which were assigned to the (100), (110), (200) and (210) planes, respectively (Fig. 2c). The broad intensity at $25.19^{\circ}$ was assigned to the (001) planes, corresponding to a layer spacing of $3.53 \AA$. Multiple reflections indicate that FS-COF has high periodicity in three dimensions. Le Bail refinements confirmed that the diffraction pattern was consistent with a primitive hexagonal lattice with unit cell parameters ( $a=b=$ 36.205(6) $\AA, c=7.285(5) \AA$ ) similar to an idealized eclipsed model of the FS-COF (Fig. 2a, upper). S-COF exhibited lower crystallinity, indicated by the broadened diffraction profile. The 
observable diffraction intensities can be accounted for by a primitive hexagonal structure with an in-plane lattice parameter of 27.44(2) $\AA$ and a $\pi$-stacking distance of approximately $3.7 \AA$. Based on both laboratory and synchrotron X-ray diffraction data (Supplementary Figs 24-27), we propose that FS-COF and S-COF have AA layer stackings (Fig. 1c), rather than AB stacking. However, there are a variety of possible AA stacking patterns (Supplementary Fig. 93) and the X-ray data do not allow us to distinguish between these (Supplementary Figs 25 and 26). For the purposes of structural comparisons with experimental data, we refer to the idealized, perfectly eclipsed AA stacking patterns (Fig, 2a,b) because properties such as porosity are not greatly affected by small shifts in the relative orientation of the layers. By contrast, calculations that are discussed later suggest that the electronic structure of FS-COF is quite strongly affected by small changes to the AA layer stacking.

The porosity of these COFs was assessed by nitrogen sorption measurements at $77.3 \mathrm{~K}$. Brunauer-Emmett-Teller (BET) surface areas of FS-COF, S-COF and TP-COF were found to be 1288,985 , and $919 \mathrm{~m}^{2} \mathrm{~g}^{-1}$. The measured surface area for FS-COF equates to $78 \%$ of the calculated nitrogen-accessible surface area for the idealized, eclipsed structure shown in Fig. 2a $\left(1652 \mathrm{~m}^{2} \mathrm{~g}^{-1}\right)$. The experimental surface areas for the two less crystalline COFs were lower than the idealized, calculated values (1690 and $2172 \mathrm{~m}^{2} \mathrm{~g}^{-1}$ for S-COF and TP-COF), although both materials were still microporous. The pore diameters derived for FS-COF, S-COF and TPCOF by fitting nonlocal density functional theory models to the $\mathrm{N}_{2}$ isotherms were $27.6 \AA, 22.8$ $\AA$ and $29.0 \AA$. All COFs gave rise to nitrogen isotherms with shapes consistent with mesoporosity and sequential, multilayer pore filling (Fig. 3a, Supplementary Figs 18-20).

HR-TEM images of FS-COF (Fig. 3b) confirm that it has an ordered, hexagonal pore structure oriented along the crystallographic $c$-axis with a periodicity of approximately $3.0 \mathrm{~nm}$ (highlighted area, Fig. 3b), which is consistent with the in-plane pore channels of $3.2 \mathrm{~nm}$ in the proposed AA-stacked COF structure. S-COF and TP-COF exhibited no such clear, ordered domains when analyzed by HR-TEM (Supplementary Fig. 31). Atomic force microscopy 
(AFM) images of FS-COF deposited onto silicon wafers from water suspensions also show that this COF can be partially exfoliated into thin stacks, albeit not single layers, with thicknesses ranging from 5-25 $\mathrm{nm}$ (Supplementary Fig. 33).

UV-visible reflectance spectra of the COFs were measured in the solid-state and the absorption onset was found to be 670, 590 and $540 \mathrm{~nm}$ for FS-COF, S-COF, and TP-COF (Fig. 4a). The onsets for FS-COF, S-COF and TP-COF are red-shifted by 70, 45 and $90 \mathrm{~nm}$ compared to their diamine monomers. The UV-visible spectra of an amorphous analogue FS-P, discussed below, shows a blue shift compared with FS-COF, but it also exhibits a red-shifted absorption compared to the diamine monomer. FS-COF absorbs more light in the visible spectrum and shows a significant red-shift in its absorption onset compared to the corresponding linear dibenzo $[b, d]$ thiophene sulfone-based polymers, P7 and P10, by 210 and $184 \mathrm{~nm}$.

\section{Photocatalysis experiments}

We next investigated the activity of these COFs for photocatalytic water reduction using ascorbic acid as a sacrificial hole-scavenger and platinum as a co-catalyst. All materials evolved hydrogen under visible light $(\lambda>420 \mathrm{~nm}$, Fig. $4 \mathrm{~b})$ and the average hydrogen evolution rates were determined to be $1.6 \mathrm{mmol} \mathrm{g}^{-1} \mathrm{~h}^{-1}$ for $\quad$ TP-COF, $4.44 \mathrm{mmol} \mathrm{g}^{-1} \mathrm{~h}^{-1}$ for S-COF, and $10.1 \mathrm{mmol} \mathrm{g}^{-1} \mathrm{~h}^{-1}$ for FS-COF. As in previous reports for porous titania glasses ${ }^{42}$, strontium titanate, ${ }^{43}$ and carbon nitride, ${ }^{5}$ we observed the production of smaller, though still significant, quantities of hydrogen without the addition of platinum for S-COF and FS-COF with rates of $0.6 \mathrm{mmol} \mathrm{g}^{-1} \mathrm{~h}^{-1}$ and $1.32 \mathrm{mmol} \mathrm{g}^{-1} \mathrm{~h}^{-1}$, respectively. No hydrogen production was observed for TP-COF without platinum. The mass-normalized hydrogen evolution rate for FSCOF of $10.1 \mathrm{mmol} \mathrm{g}^{-1} \mathrm{~h}^{-1}$ is the highest reported for a photocatalytically active COF (Table 1). This rate is 22 times higher than for $\mathbf{N}_{\mathbf{3}}$ - $\mathbf{C O F}\left(0.47 \mathrm{mmol} \mathrm{g}^{-1} \mathrm{~h}^{-1}\right)$, as measured by us under identical conditions over 5 hours (with ascorbic acid), and around six times higher than the optimized rate reported for $\mathbf{N}_{3}-\mathbf{C O F}$ by Vyas et al. using triethanolamine as a sacrificial donor. ${ }^{35}$ 
An external quantum efficiency (EQE) of 3.2\% was determined for FS-COF at $420 \mathrm{~nm}$ (violet light), whereas $1.3 \%$ was reported for the diacetylene $\mathrm{COF}^{37}$ at $420 \mathrm{~nm}$ and $0.44 \%$ for $\mathbf{N}_{3}-\mathbf{C O F}$ at $450 \mathrm{~nm}$ both using TEOA as a scavenger. ${ }^{35}$ At even longer-wavelengths $(600 \mathrm{~nm}$, orange light), FS-COF still displayed an EQE of 0.6\% (Supplementary Fig. 40).

Longer-term photolysis experiments for FS-COF with up to 50 hours of visible light irradiation $(\lambda>420 \mathrm{~nm}$, Fig. 4c) showed no significant decrease in the catalytic performance over time, suggesting good stability. No changes to the PXRD patterns were observed after long-term irradiation, showing that the crystallinity was retained (Supplementary Fig. 49). For comparison, we estimate that the 50 hour photolysis reported for $\mathbf{N}_{3}-\mathbf{C O F},{ }^{35}$ also using ascorbic acid as a sacrificial donor, yielded an average hydrogen evolution rate of around $0.08 \mathrm{mmol} \mathrm{g}^{-}$ ${ }^{1} \mathrm{~h}^{-1}$ (Supplementary Fig. 63 in ref. 35); about 125 times lower than we observe for FS-COF.

The marked difference in catalytic activity between FS-COF and the isostructural framework TP-COF can be explained at least in part by the red-shift in the absorption onset, which allows FS-COF to absorb more visible photons. The partial exfoliation of FS-COF may also contribute. ${ }^{10,44}$ The higher BET surface area of $1288 \mathrm{~m}^{2} \mathrm{~g}^{-1}$ for FS-COF versus $919 \mathrm{~m}^{2} \mathrm{~g}^{-1}$ for TP-COF might also enhance the availabliity of photogenerated charges for water reduction. For context, diffusion lengths of up to around $10 \mathrm{~nm}$ have been reported for thin-films of conjugated polymers; ${ }^{45-47}$ hence, photogenerated charges produced inside non-porous particles much larger than $10 \mathrm{~nm}$ may not reach the particle surface.

Introducing sulfone groups results in much lower contact angles with pure water for FS-COF $\left(23.6^{\circ}\right)$ and S-COF $\left(43.7^{\circ}\right)$ in comparison with TP-COF $\left(59.7^{\circ}\right)$ and $\mathbf{N}_{3}-\mathbf{C O F}\left(53.4^{\circ}\right)$. These contact angles are low for organic materials: for reference, most organic polymers have contact angles in the range $60-110^{\circ}$, and poly(vinyl alcohol) has a contact angle of around $51^{\circ} .48$ Water vapour uptake measurements (Fig. 3c) show type II isotherms for FS-COF, S-COF and TP-COF. Functionalized FS-COF and S-COF adsorb 67 wt. \% and 42 wt. \% water at $293 \mathrm{~K}$ and 22.9 mbar; by comparison, TP-COF adsorbs only $16 \mathrm{wt}$. \% water under the same 
conditions. This is due to water condensation ${ }^{49}$ within the mesopores of FS-COF, which are decorated with a large number of polarized heteroatoms (Fig. 2a). Wetting matters in aqueous photocatalysis because particle dispersibility and favourable interactions with water and the sacrificial donor are required for good photocatalytic performance. The water isotherm for FSCOF shows that the internal pore structure of the material is accessible to water, as well as the external surface, thus increasing the number of potential sites for photocatalytic water reduction.

Besides light absorption, particle size, and wettability, crystallinity might be important in the catalytic the performance of FS-COF. In particular, the eclipsed AA layered structure of FSCOF (Fig. 2a) might facilitate charge-carrier transport in the material, although it is not possible at this stage to deconvolute this from other factors, such as surface area. Increased long-range order in carbon nitride was reported to improve photocatalytic activity by enhancing chargetransport to active sites. ${ }^{22}$ In this study, both S-COF and FS-COF strongly outperformed the corresponding semi-crystalline conjugated co-polymers, $\mathbf{P} 7^{16}$ and $\mathbf{P 1 0}$, that were the inspiration for these frameworks (Fig. 1, Table 1): under comparable conditions, FS-COF is around twelve times more active than $\mathbf{P 7}$, and it is seven times more active than $\mathbf{P 1 0}$ (Table 1).

To further investigate the effect of crystallinity on photocatalytic performance, we synthesized FS-P, an almost amorphous analogue of FS-COF (Supplementary Fig. 29). We did this by carrying out the synthesis using 1,2-dichlorobenzene as solvent, rather than mixture of 1,4dioxane and mesitylene (Supplementary Section 2). Amorphous FS-P showed much lower photocatalytic activity $(\lambda>420 \mathrm{~nm})$ and a hydrogen evolution rate of only $1.12 \mathrm{mmol} \mathrm{g}^{-1} \mathrm{~h}^{-1}$; nine times lower than FS-COF. FS-P showed a slightly blue-shifted absorption on-set (Fig. 4a) relative to FS-COF, possibly because the delocalization that arises from $\pi-\pi$ stacking in FSCOF is disrupted in this amorphous analogue, although its visible light absorption profile is still more favourable than S-COF or TP-COF. The low activity of FS-P may also be related to 
its reduced surface area $\left(209 \mathrm{~m}^{2} \mathrm{~g}^{-1}\right)$ and a lower degree of condensation in the amorphous polymer. Weak absorption bands were observed in the FT-IR spectrum at 3371 and $3473 \mathrm{~cm}^{-1}$, which are probably due to amine end-groups. ${ }^{29}$ Another important factor could be the particle size of the in situ deposited platinum co-catalyst: FS-COF has well-defined $3 \mathrm{~nm}$ sized platinum nanoparticles on its surface (Supplementary Fig. 32), and it is possible that the size is controlled by the uniform mesopores in the COF. By contrast, undefined micrometer-sized platinum aggregates were formed on the surface of amorphous FS-P.

We used time-correlated single photon counting to estimate the excited state lifetimes for these materials in aqueous suspensions (Fig. 4d). The average weighted lifetime of FS-COF ( $\tau_{\text {avg }}=$ $5.56 \mathrm{~ns})$ was estimated to be significantly longer than that of TP-COF ( $\left.\tau_{\text {avg }}=0.25 \mathrm{~ns}\right)$ or FS-P $\left(\tau_{\text {avg }}=2.21 \mathrm{~ns}\right)$, which correlates with the higher photocatalytic performance observed for FS-

\section{COF.}

\section{Computational studies}

For a COF to act as a hydrogen evolution photocatalyst, it must absorb light efficiently over a broad range in the visible spectrum as well transporting the electron-hole pairs, or excitons, that are formed upon light absorption. The COF must also thermodynamically drive the reduction of protons and the oxidation of water — or in this study, the sacrificial electron donor (SED), ascorbic acid. To achieve this, the electron affinity (EA) of the COF or its exciton ionization potential (IP*) and the ionization potential (IP) of the COF or its exciton electron affinity $\left(\mathrm{EA}^{*}\right)$ should straddle the proton reduction and water/SED oxidation potentials. ${ }^{50}$ Because the potentials of a polymer are difficult to measure experimentally,${ }^{51}$ we instead predicted them computationally using density functional theory (DFT). We did this using two different approaches; cluster calculations on representative fragments of the COFs embedded in a dielectric continuum to model the $\mathrm{COF}$-water interface and periodic calculations on $\mathrm{COF}$ crystal structures. These two approaches complement each other. By necessity, the periodic calculations approximate IP and EA by the Kohn-Sham valence band maximum (VBM) and 
conduction band minimum (CBM). Hence, these calculations cannot describe the effect of immersing the COF in water, but they do take into account the influence of layer stacking. Cluster calculations are limited to a fragment, but unlike the periodic calculations, they describe the effect of water and allow us to calculate the exciton potentials and the exciton binding energy (EBE). The latter is important for polymers, where the EBE is generally large relative to $k \mathrm{~T}$ (26 meV at room temperature) such that spontaneous dissociation of excitons into free electrons and holes is unlikely.

The cluster DFT (B3LYP) calculations on fragments embedded in a continuum with the relative dielectric permittivity of water predict that S-COF, FS-COF and TP-COF should all have a substantial thermodynamic driving forces for proton reduction (Fig. 5a,b). Water oxidation is predicted to be endergonic or negligibly exergonic, providing a thermodynamic explanation for the inability of these materials to drive hydrogen evolution without a sacrificial agent. Ascorbic acid was used as a SED because its one-hole and two-hole oxidation potentials are more negative than the water oxidation potential, meaning it is more easily oxidized (Fig. 5a).

Cluster calculations for $\mathbf{N}_{3}$-COF (Fig. 5a,b) suggest a different picture. While the IP and EA of $\mathrm{N}_{3}$ (L) straddle both water splitting half-reaction potentials, suggesting a driving force for overall water splitting, the EA* and IP* do not; this means that overall water splitting is still not thermodynamically favoured without bulk exciton dissociation — and the exciton binding energy is predicted to be as large as $0.92 \mathrm{eV}$. Calculations using range-separated density functionals also suggest that the exciton binding energy in $\mathbf{N}_{3}-\mathbf{C O F}$ is large (Supplementary Fig. 91). The IP* of $N_{3}(L)$ is only marginally more negative than the potential of proton reduction and the EA* marginally more positive than the potential of the one-hole oxidation of ascorbic acid. The relatively low photocatalytic activity of $\mathbf{N}_{3}-\mathbf{C O F}$ with ascorbic acid might therefore be linked to the combined effects of difficult exciton dissociation in the bulk material and the small driving forces associated with exciton dissociation at the photocatalyst-solution interface. 
Next, periodic DFT (HSE06) calculations were performed to investigate the electronic structures of the COF crystals. The position of the VBM and CBM for each COF crystal structure was referenced to a common vacuum level (Fig. 5c), determined by the value of the electrostatic potential at the centre of an internal pore. Both eclipsed (AA) and staggered (AB) stacking sequences were considered for S-COF, FS-COF, and TP-COF (Fig. 5d); the eclipsed AA layered structure of $\mathbf{N}_{3}-\mathbf{C O F}$ was also included for comparison. Our periodic results corroborate the molecular fragment picture; all of these COF materials are predicted, in certain AA packings, to be thermodynamically able to reduce protons and oxidize ascorbic acid, in line with experiments. The exact positions of VBM and CBM for FS-COF were found to be sensitive to the small changes to the crystal structure, and this is most likely true for the other COFs as well. The CBM of the idealized, eclipsed AA-stacked FS-COF structure lies, in fact, below the proton reduction potential: hence, in the absence of water at least, this structure is not predicted to drive proton reduction. However, calculations show that minor levels of disorder, such as small offsets between neighbouring layers or partial/full flipping of the FS linkers in alternating layers, can alter the band edge positions (black horizontal lines in Fig. 5c, also Supplementary Fig. 93). All of these possible AA-stacked structure models are close in computed total lattice energy (Supplementary Table 7) and we cannot distinguish between them using either laboratory or synchrotron PXRD data (Supplementary Fig. 26). These calculations, coupled with the photocatalytic proton reduction observed for FS-COF, suggest that the crystal packing might not be exclusively the idealized AA stacking, although we note that the effect of water, as discussed above, cannot be included in these periodic calculations.

As observed experimentally, calculations predict correctly that FS-COF has the smallest optical gap among the four COF materials studied here and thus, probably, the largest rates of visible photon absorption and exciton generation. This is combined with favourably positioned IP/EA*/VBM and EA/IP*/CBM levels, at least for certain AA packings, to sustain a driving force for both redox half reactions. Other factors may also contribute to the photocatalytic performance of FS-COF, such as its strong affinity for water (Fig. 3c), good wettability 
(Supplementary Fig. 34), well-dispersed Pt cocatalyst particles (Supplementary Fig. 32), and the longer excited state lifetime in this material (Fig. 4d).

\section{Dye sensitization}

The ordered mesoporosity in these frameworks offers various opportunities for post-synthetic modification. For example, we explored the dye sensitization of FS-COF with the goal of further enhancing its photocatalytic performance. ${ }^{52}$ Addition of $2^{\prime}, 7^{\prime}$-dichlorofluorescein (Supplementary Fig. 75) reduced the photocatalytic performance of FS-COF, while Rose Bengal (sodium salt of 4,5,6,7-tetrachloro-2',4',5',7'-tetraiodofluorescein) slightly improved the performance. However, when Eosin $\mathrm{Y}\left(2^{\prime}, 4^{\prime}, 5^{\prime}, 7^{\prime}\right.$-tetrabromofluorescein) was added, hydrogen evolution rates for FS-COF were enhanced by up to $60 \%$; from $10.1 \mathrm{mmol} \mathrm{g}^{-1} \mathrm{~h}^{-1}$ to $13 \mathrm{mmol} \mathrm{g}^{-1} \mathrm{~h}^{-1}\left(10 \mathrm{mg}\right.$ dye, $5 \mathrm{mg}$ of FS-COF) and to $16.1 \mathrm{mmol} \mathrm{g}^{-1} \mathrm{~h}^{-1}$ when $20 \mathrm{mg}$ of the dye was added. Further increases in the dye loading reduced the catalytic rate (Supplementary Fig. 76). By contrast, the hydrogen evolution rate of $5 \mathrm{mg}$ of amorphous FS-P was reduced from $1.1 \mathrm{mmol} \mathrm{g}^{-1} \mathrm{~h}^{-1}$ to $0.58 \mathrm{mmol} \mathrm{g}^{-1} \mathrm{~h}^{-1}$ in the presence of $20 \mathrm{mg}$ of Eosin $\mathrm{Y}$. The absorption spectrum of Eosin Y overlaps with the absorption spectrum of FS-COF, so the addition of dye enhances the total absorption cross section of the system. Likewise, when a larger amount of FS-COF was used with no dye, then a similar increase in the hydrogen evolution rate was observed (12.9 $\mathrm{mmol} \mathrm{g}^{-1} \mathrm{~h}^{-1}$ for $10 \mathrm{mg}$ FS-COF versus $10.1 \mathrm{mmol} \mathrm{g}^{-1} \mathrm{~h}^{-1}$ for $5 \mathrm{mg}$ FS-COF).

Eosin Y has a similar absorption spectrum to FS-COF, so it does not harvest additional photons in the energy-rich near-infrared region of the solar spectrum where FS-COF does not absorb. A greater enhancement in the hydrogen evolution rate was observed when a near-infrared absorbing dye, WS5F, was used. Unlike the previous dyes, WS5F does not dissolve well in water so it was pre-loaded into the COF using acetone before the photocatalytic tests (loading conditions: $5 \mathrm{mg}$ FS-COF $+5 \mathrm{mg}$ WS5F). When $5 \mathrm{mg}$ of this dye-sensitized material, FSCOF+WS5F was used, we observed a visible light hydrogen evolution rate of $16.3 \mathrm{mmol} \mathrm{g}$ -

${ }^{1} \mathrm{~h}^{-1}$, again normalized to the mass of the COF. We ascribe this enhancement to the absorption 
of more photons at higher wavelengths by the dye-loaded composite, as expressed in the EQE at 600 and $700 \mathrm{~nm}$ when using monochromatic light. At $600 \mathrm{~nm}$, FS-COF has an EQE of 0.6\%, which is increased to $2.2 \%$ for the FS-COF+WS5F composite. At $700 \mathrm{~nm}$, the composite has an EQE of 0.7\%, while FS-COF is completely inactive.

We attribute the dye-sensitization effect to host-guest interactions in the large, hydrophilic 1-D mesopore channels (Supplementary Fig. 94), which may help to explain why dye sensitization with Eosin Y and WS5F was unsuccessful for the less porous FS-P material. Control experiments using the neat dyes, Eosin Y and WS5F, in the absence of FS-COF showed negligible hydrogen production under visible light ( $0.1 \mathrm{M}$ ascorbic acid solution plus platinum). Likewise, WS5F supported on mesoporous silica (SBA-15) showed no hydrogen evolution under the same conditions.

The photoluminescence (PL) spectra of FS-COF+WS5F were measured to investigate the interaction between FS-COF and WS5F. As shown in Supplementary Fig. 70, WS5F solution in acetone excited at $410 \mathrm{~nm}$ exhibits an intense emission peak at $630 \mathrm{~nm}$, which can be quenched by increasing the concentration of colloidal FS-COF. Since there is no obvious overlap between the absorption spectrum of FS-COF and the PL emission of WS5F, the fluorescence quenching in WS5F+FS-COF is likely to be the result of interfacial electron transfer from the excited dye to FS-COF. This is supported by calculations that suggest a favorable energy alignment (Fig. 6d): for example, one possible scheme is that the photoexcited dye transfers electrons to FS-COF and is then regenerated by the sacrificial electron donor. ${ }^{53}$

\section{Photocatalysis experiments with thin COF films}

Sacrificial hydrogen production is a step on the path to overall water splitting, but the latter may require the construction of more sophisticated architectures, such as Z-schemes. ${ }^{54-56} \mathrm{Z}$-schemes comprise two separate, coupled semiconductor phases, where each phase carries out one of the half-reactions in overall water splitting. To create such architectures, processability is important. ${ }^{57}$ We found that FS-COF can be dispersed as a colloidal solution in various solvents 
(Fig. 6f), and we used this to drop-cast platinized FS-COF onto glass supports. Photocatalytic hydrogen evolution rates ( $\lambda>420 \mathrm{~nm}, 0.1 \mathrm{M}$ ascorbic acid, 5 hours irradiation) were found to increase with the number of drop-casting cycles (Supplementary Fig. 84), presumably due to increased film thickness, up to $24.9 \mathrm{mmol} \mathrm{h}^{-1} \mathrm{~m}^{-2}$ after 20 successive depositions of the colloidal solution. Longer-term hydrogen evolution experiments for a COF film produced with just a single drop-cast cycle showed steady hydrogen production over 20 hours, indicating that the film was stable under the reaction condition $(\lambda>420 \mathrm{~nm}, 0.1 \mathrm{M}$ ascorbic acid) (Figure 6e). We also tested hydrogen evolution of this film under solar simulator irradiation (AM1.5G, classification ABA, ASTM E927-10), which gave a hydrogen evolution rate of $15.8 \mathrm{mmol} \mathrm{h}^{-1}$ $\mathrm{m}^{-2}\left(\sim 0.36 \mathrm{~L} \mathrm{~h}^{-1} \mathrm{~m}^{-2}\right)$. This can be compared with data obtained at the lab-scale for carbon nitride films (0.19 $\left.\mathrm{L} \mathrm{h}^{-1} \mathrm{~m}^{-2}\right){ }^{58}$ Scanning electron micrographs (Supplementary Fig. 81) show that these COF films had smooth, uniform morphologies, and AFM analysis (Supplementary Fig. 82) indicates that the film after one drop-casting cycle is around $10 \mathrm{~nm}$ thick.

\section{CONCLUSIONS}

Although crystallinity is not required for all applications of porous materials,${ }^{59}$ here we see a dramatic enhancement in photocatalytic hydrogen evolution rates for ordered, crystalline COFs over structurally-related amorphous or semi-crystalline solids. Organic building blocks that function well in amorphous polymers, such as dibenzo $[b, d]$ thiophene sulfone, lead to materials with better catalytic function when incorporated into COFs. A fused building block, benzo[1,2$\left.b: 4,5-b^{\prime}\right]$ bis $[b]$ benzothiophene sulfone, forms a COF with a sacrificial hydrogen evolution rate that exceeds our best linear polymers, $\mathbf{P} 7$ and $\mathbf{P 1 0}$ under comparable conditions, and that is also higher than other reported COFs. ${ }^{32-37}$ Because FS-COF is mesoporous, it can be dye sensitized to give even higher hydrogen evolution rates of up to $16.3 \mathrm{mmol} \mathrm{g}^{-1} \mathrm{~h}^{-1}$. FS-COF is also stable for at least 50 hours of photolysis in water under visible light $(\lambda>420 \mathrm{~nm})$, and can be cast as a colloid onto planar supports to form thin films while still retaining its photocatalytic activity and stability.

Computation suggests that the fine detail of the AA layer stacking in FS-COF, and by analogy other COF materials, may determine the prospects for thermodynamic proton reduction and 
water oxidation. To improve our understanding of structure-property relationships, it would be helpful to produce COFs with greater degrees of long-range order. Recent synthetic developments, such as seeded growth strategies, ${ }^{60}$ offer one way forward.

Proton reduction using a sacrificial electron donor is only the first step toward full water splitting ${ }^{61}$ but the mesoporous morphology of these COFs, their processability into films, and their high native photocatalytic activity makes them attractive platforms for developing hybrid photocatalysts. For example, the internal pore structure of COFs such as FS-COF could be decorated with quantum dots, photoactive organic molecules, fullerenes, or single-site molecular catalysts. COFs with even larger mesopores might be designed to accommodate a second organic or inorganic semiconductor in the pore channels to produce a Z-scheme photocatalyst for overall water splitting. ${ }^{62}$

\section{METHODS}

COF Synthesis. All COFs were prepared using a procedure based on the method described here for the synthesis of FS-COF. A Pyrex tube was charged with 2,4,6-triformylphloroglucinol (10.5 mg, $0.05 \mathrm{mmol}$ ), 3,9-diamino-benzo[1,2-b:4,5- $\left.b^{\prime}\right]$ bis[1] benzothiophene-5,5,11,11tetraoxide $(28.8 \mathrm{mg}, 0.075 \mathrm{mmol})$, mesitylene $(1.5 \mathrm{~mL}), 1,4$-dioxane $(1.5 \mathrm{~mL})$, and aqueous acetic acid $(0.3 \mathrm{~mL}, 6 \mathrm{M})$. This mixture was homogenized by sonication for 10 minutes and the tube was then flash frozen at $77.3 \mathrm{~K}$ (liquid $\mathrm{N}_{2}$ bath) and degassed by three freeze-pump-thaw cycles, before evacuating to a pressure of 100 mTorr. The tube was sealed and then heated at $120^{\circ} \mathrm{C}$ for 3 days. The brown precipitate was collected by centrifugation and washed with $N, N-$ dimethylformamide $(100 \mathrm{~mL})$ and acetone $(200 \mathrm{~mL})$. After drying at $120^{\circ} \mathrm{C}$, the product was obtained a deep red powder (21 mg, 58\%). Anal. Calcd for $\left(\mathrm{C}_{30} \mathrm{H}_{22} \mathrm{~N}_{2} \mathrm{O}_{8} \mathrm{~S}_{2}\right)_{n}$ : C, 61.42; H, 3.78; N, 4.78; S, 10.93. Found: C, 44.80; H, 3.21; N, 3.95; S, 9.93.

Dye sensitization. For water insoluble dyes such as WS5F, the dye was loaded into the COF using an organic solvent prior to hydrogen evolution experiments. To do this, $5 \mathrm{mg}$ WS5F was dissolved in $10 \mathrm{~mL}$ acetone and then $5 \mathrm{mg}$ FS-COF was added to the solution and stirred for 12 hours. The resulting mixture was filtered, and the filtrate was dried at $80{ }^{\circ} \mathrm{C}$ overnight. Amorphous FS-P was loaded with WS5F in the same way. For water-soluble dyes, the dye was added directly into the photocatalytic mixture. In a typical procedure, a flask was charged with 
COF powder (or amorphous polymer) (5 mg), aqueous $0.1 \mathrm{M}$ ascorbic acid solution $(25 \mathrm{~mL})$, and hexachloroplatinic acid ( $5 \mu \mathrm{L}, 8 \mathrm{wt}$. \% aqueous solution). In the case of dye sensitization experiments with water-soluble dyes (Eosin $\mathrm{Y}\left(2^{\prime}, 4^{\prime}, 5^{\prime}, 7^{\prime}\right.$-tetrabromofluorescein), 2' ,7' -dichlorofluorescein or Rose Bengal), the dye was added directly to the flask. Hydrogen evolution rates were normalized to the mass of the $\mathrm{COF}$ (or amorphous polymer) in all cases.

Hydrogen evolution experiments. A flask was charged with the photocatalyst powder ( $5 \mathrm{mg}$ ), $0.1 \mathrm{M}$ ascorbic acid water solution $(25 \mathrm{~mL})$, hexachloroplatinic acid $(5 \mu 1,8$ wt. \% aqueous solution) as a platinum precursor and water-soluble dye (if any). The resulting suspension was ultrasonicated for 20 minutes before degassing by $\mathrm{N}_{2}$ bubbling for 30 minutes. The reaction mixture was illuminated with a $300 \mathrm{~W}$ Newport Xe light-source (Model: 6258, Ozone free) for the period specified using appropriate filters. The lamp was cooled by water circulating through a metal jacket. Gas samples were taken with a gas-tight syringe and analysed using a Bruker 450-GC gas chromatograph. Hydrogen was detected with a thermal conductivity S3 detector referencing against a standard gas of known concentration. Hydrogen dissolved in the reaction mixture was not measured and the pressure increase generated by the evolved hydrogen was neglected in the calculations. The rates were determined from a linear regression fit. After 5 hours of photocatalysis, no carbon monoxide associated with framework or scavenger decomposition could be detected on a GC system equipped with a pulsed discharge detector. After the photocatalysis experiment, the FS-COF was recovered by washing with water and acetone before drying at $120^{\circ} \mathrm{C}$.

Calculations. For the different molecular fragments representing the COFs, we calculated the standard reduction potentials of half-reactions for free electrons/holes and excitons, using density functional theory (DFT) and time-dependent DFT (TD-DFT). The B3LYP density functional was used for all the DFT and TD-DFT calculations (unless otherwise stated), together with the Def2-SVP basis set, ${ }^{63}$ using the Gaussian 16 software. ${ }^{64} \mathrm{~S} 1$ optimizations for the calculations of the exciton potentials (i.e., IP* and EA*) used the Tamm-Dancoff approximation. ${ }^{65}$ The effect of solvation by water was accounted for by using the PCM/SMD solvation model. ${ }^{66,67}$ The potentials of the solution reactions for one-hole and two-hole oxidation of ascorbic acid were calculated as described in Supplementary section 22, while the experimental values were used for the proton reduction and water oxidation reactions.

Periodic DFT calculations on the COF crystal structures were carried out within the plane-wave pseudopotential formalism, using the Vienna ab initio Simulation Package (VASP) code. ${ }^{69}$ Geometry optimizations were performed employing the Perdew-Burke-Ernzerhof exchange-correlation functional with the DFT-D3(BJ) dispersion correction. ${ }^{70-72}$ A kinetic- 
energy cutoff of $500 \mathrm{eV}$ was used to define the plane-wave basis set. The electronic structure of each optimized COF structure was then computed using a screened hybrid exchange-correlation functional (HSE06) ${ }^{73-75}$ giving key electronic properties, such as band gap and electrostatic potential. To achieve valence band alignment so that band energies can be compared for the different COF structures, we followed an approach devised for determining the vacuum level of porous structures. ${ }^{76}$ Further computational details are provided in Supplementary section 22 .

Data availability. All data generated or analysed during this study are included in this published article and the supplementary information files.

\section{Additional information}

Supplementary information is available in the online version of the paper. Reprints and permissions information is available online at www.nature.com/reprints. Correspondence and requests for materials should be addressed to A.I.C.

\section{Author contributions}

A.I.C. and X.W. conceived the project. X.W. synthesized the COFs and performed the characterization and photocatalysis experiments. L.C. and M.A.Z. conceived the modelling strategy and performed the calculations. S.Y.C. carried out powder X-ray diffraction analyses. M.A.L. carried out single-crystal X-ray structure analysis. R.S.S. performed the TSPC experiments and cosupervised, with A.I.C., the work on COF synthesis, characterization, and photocatalysis. Y. Y. collected the water sorption isotherms. R.C., X.W. and L.C. interpreted the gas sorption isotherms. Y. W. and W.-H. Z. synthesized and characterized the WS5F dye. All authors interpreted the data and contributed to the preparation of the manuscript.

\section{Competing financial interests}

The authors declare no competing financial interest. 


\section{Acknowledgements}

We acknowledge funding from the Engineering and Physical Sciences Research Council (EPSRC) (EP/N004884/1), the European Union's Seventh Framework Programme through grant agreement numbers 321156 (ERC-AG-PE5-ROBOT) and 692685, and the Leverhulme Trust via the Leverhulme Research Centre for Functional Materials Design. X.W. thanks the China Scholarship Council for a Ph.D. studentship. Y. W. and W.-H. Z. acknowledge financial support from NSFC for Creative Research Groups (21421004) and Key Project (21636002), NSFC/China and Shanghai Oriental Scholarship. We thank Matthew Bilton for help with HRTEM, Fiona McBride for help with AFM, Guo-Hong Ning and Hongjun Niu for useful discussions. We thank Diamond Light Source for access to beamlines I19 (MT15777) and I11 (EE12336). We acknowledge the ARCHER UK National Supercomputing Service, access provided via a Programme Grant (EP/N004884) and the EPSRC funded UK Materials Chemistry Consortium (EP/L000202/1), and the use of the facilities of N8 HPC Centre of Excellence, provided and funded by the N8 Research Partnership and EPSRC (EP/K000225/1).

\section{REFERENCES}

1. Fujishima, A. \& Honda, K. Electrochemical photolysis of water at a semiconductor electrode. Nature 238, 37-38 (1972).

2. Kudo, A. \& Miseki, Y. Heterogeneous photocatalyst materials for water splitting. Chem. Soc. Rev. 38, 253-278 (2009).

3. Sivula, K. \& van de Krol, R. Semiconducting materials for photoelectrochemical energy conversion. Nat. Rev. Mater. 1, 15010 (2016).

4. Chen, S., Takata, T. \& Domen, K. Particulate photocatalysts for overall water splitting. Nat. Rev. Mater. 2, 17050 (2017).

5. Wang, X. et al. A metal-free polymeric photocatalyst for hydrogen production from 
water under visible light. Nat. Mater. 8, 76-80 (2009).

6. Sprick, R. S. et al. Tunable organic photocatalysts for visible-light-driven hydrogen evolution. J. Am. Chem. Soc. 137, 3265-3270 (2015).

7. Zhang, G., Lan, Z.-A. \& Wang, X. Conjugated polymers: catalysts for photocatalytic hydrogen evolution. Angew. Chem. Int. Ed. 55, 2-18 (2016).

8. Yanagida, S., Kabumoto, A., Mizumoto, K., Pac, C. \& Yoshino, K. Poly(para)phenylene-catalyzed photoreduction of water to hydrogen. JCS-Chem. Commun. 8, 474-475 (1985).

9. Shibata, T. et al. Novel visible-light-driven photocatalyst. poly(p-phenylene)-catalyzed photoreductions of water, carbonyl compounds, and olefins. J. Phys. Chem. 94, 2068$2076(1990)$.

10. Schwinghammer, K. et al. Crystalline carbon nitride nanosheets for improved visiblelight hydrogen evolution. J. Am. Chem. Soc. 136, 1730-1733 (2014).

11. Schwab, M. G. et al. Photocatalytic hydrogen evolution through fully conjugated poly(azomethine) networks. Chem. Commun. 46, 8932 (2010).

12. Yang, C. et al. Molecular engineering of conjugated polybenzothiadiazoles for enhanced hydrogen production by photosynthesis. Angew. Chem. Int. Ed. 55, 9202-9206 (2016).

13. Li, L. et al. Rational design of porous conjugated polymers and roles of residual palladium for photocatalytic hydrogen production. J. Am. Chem. Soc. 138, 7681-7686 (2016).

14. Woods, D. J., Sprick, R. S., Smith, C. L., Cowan, A. J. \& Cooper, A. I. A solutionprocessable polymer photocatalyst for hydrogen evolution from water. Adv. Energy Mater. 7, 1700479 (2017).

15. Sprick, R. S. et al. Extended conjugated microporous polymers for photocatalytic 
hydrogen evolution from water. Chem. Commun. 52, 10008-10011 (2016).

16. Sprick, R. S. et al. Visible-light-driven hydrogen evolution using planarized conjugated polymer photocatalysts. Angew. Chem. Int. Ed. 55, 1792-1796 (2016).

17. Wang, k. et al. Covalent triazine frameworks via a low temperature polycondensation approach. Angew. Chem. Int. Ed. 56, 14337-14341 (2017).

18. Meier, C. B. et al. Structure-property relationships for covalent triazine-based frameworks: the effect of spacer length on photocatalytic hydrogen evolution from water. Polymer. 126, 283-290 (2017).

19. Bi, J. et al. Covalent triazine-based frameworks as visible light photocatalysts for the splitting of water. Macromol. Rapid Commun. 36, 1799-1805 (2015).

20. Zhang, G., Lan, Z.-A., Lin, L., Lin, S. \& Wang, X. Overall water splitting by Pt/g- $\mathrm{C}_{3} \mathrm{~N}_{4}$ photocatalysts without using sacrificial agents. Chem. Sci. 7, 3062-3066 (2016).

21. Wang, L. et al. Conjugated microporous polymer nanosheets for overall water splitting using visible light. Adv. Mater. 29, 1702428 (2017).

22. Zhang, G. et al. Optimizing optical absorption, exciton dissociation, and charge transfer of a polymeric carbon nitride with ultrahigh solar hydrogen production activity. Angew. Chem. Int. Ed. 56, 13445-13449 (2017).

23. Coropceanu, V. et al. Charge transport in organic semiconductors. Chem. Rev. 107, 926952 (2007)

24. Ockwig, N. W., Cote, A. P., Keeffe, M. O., Matzger, A. J. \& Yaghi, O. M. Porous, crystalline, covalent organic frameworks. Science 310, 1166-1171 (2005).

25. El-Kaderi, H. M. et al. Designed synthesis of 3D covalent organic frameworks. Science 316, 268-272 (2007). 
26. Diercks, C. S. \& Yaghi, O. M. The atom, the molecule, and the covalent organic framework. Science 355, eaal1585 (2017).

27. Spitler, E. L. et al. Lattice expansion of highly oriented 2D phthalocyanine covalent organic framework films. Angew. Chem. Int. Ed. 51, 2623-2627 (2012).

28. Spitler, E. L. \& Dichtel, W. R. Lewis acid-catalysed formation of two-dimensional phthalocyanine covalent organic frameworks. Nat. Chem. 2, 672-677 (2010).

29. Kandambeth, S. et al. Construction of crystalline 2D covalent organic frameworks with remarkable chemical (acid/base) stability via a combined reversible and irreversible route. J. Am. Chem. Soc. 134, 19524-19527 (2012).

30. Huang, N., Wang, P. \& Jiang, D. Covalent organic frameworks: a materials platform for structural and functional designs. Nat. Rev. Mater. 1, 16068 (2016).

31. Wan, S. et al. Covalent organic frameworks with high charge carrier mobility. Chem. Mater. 23, 4094-4097 (2011).

32. Thote, J. et al. A covalent organic framework-cadmium sulfide hybrid as a prototype photocatalyst for visible-light-driven hydrogen production. Chem. Eur. J. 20, 1596115965 (2014).

33. Zhou, J. et al. A (001) dominated conjugated polymer with high-performance of hydrogen evolution under solar light irradiation. Chem. Commun. 53, 10536-10539 (2017).

34. Stegbauer, L., Schwinghammer, K. \& Lotsch, B. V. A hydrazone-based covalent organic framework for photocatalytic hydrogen production. Chem. Sci. 5, 2789-2793 (2014).

35. Vyas, V. S. et al. A tunable azine covalent organic framework platform for visible lightinduced hydrogen generation. Nat. Commun. 6, 8508 (2015).

36. Haase, F. et al. Structure-property-activity relationships in a pyridine containing azine- 
linked covalent organic framework for photocatalytic hydrogen evolution. Faraday Discuss. 162, 165-169 (2017).

37. Pachfule, P. et al. Diacetylene functionalized covalent organic framework (COF) for photocatalytic hydrogen generation. J. Am. Chem. Soc. 140, 1423-1427 (2018).

38. Banerjee, T. et al. Single site photocatalytic $\mathrm{H}_{2}$ evolution from covalent organic frameworks with molecular cobaloxime Co-catalysts. J. Am. Chem. Soc. 139, 16228 16234 (2017).

39. Lin, S. et al. Covalent organic frameworks comprising cobalt porphyrins for catalytic $\mathrm{CO}_{2}$ reduction in water. Science 349, 1208-1213 (2015).

40. Sick, T. et al. Oriented films of conjugated 2D covalent organic frameworks as photocathodes for water splitting. J. Am. Chem. Soc. 140, 2085-2092 (2018).

41. Zhu, Y. \& Zhang, W. Reversible tuning of pore size and $\mathrm{CO}_{2}$ adsorption in azobenzene functionalized porous organic polymers. Chem. Sci. 5, 4957-4961 (2014).

42. Kruczynski, L. et al. Porous titania glass as a photocatalyst for hydrogen production from water. Nature 291, 399-401 (1981).

43. Wagner, F. T. \& Somorjai, G. A. Photocatalytic hydrogen production from water on Ptfree $\mathrm{SrTiO}_{3}$ in alkali hydroxide solutions. Nature 285, 559-560 (1980).

44. Corp, K. L., Schlenker, C. W., Corp, K. L. \& Schlenker, C. W. Ultrafast spectroscopy reveals electron transfer cascade that improves hydrogen evolution with carbon nitride photocatalysts. J. Am. Chem. Soc. 139, 7904-7912 (2017).

45. Kroeze, J. E., Savenije, T. J., Vermeulen, M. J. W. \& Warman, J. M. Contactless determination of the photoconductivity action spectrum, exciton diffusion length, and charge separation efficiency in polythiophene-sensitized $\mathrm{TiO}_{2}$ bilayers. J. Phys. Chem. $B$ 107, 7696-7705 (2003). 
46. Bruno, A., Reynolds, L. X., Dyer-Smith, C., Nelson, J. \& Haque, S. A. Determining the exciton diffusion length in a polyfluorene from ultrafast fluorescence measurements of polymer/fullerene blend films. J. Phys. Chem. C 117, 19832-19838 (2013).

47. Shaw, P. E., Ruseckas, A. \& Samuel, I. D. W. Exciton diffusion measurements in poly(3hexylthiophene). Adv. Mater. 20, 3516-3520 (2008).

48. Tezuka, Y., Fukushima, A., Matsui, S., \& Imai, K. J. Colloid Interface Sci. 114, 16-25 (1986)

49. Biswal, B. P. et al. Pore surface engineering in porous, chemically stable covalent organic frameworks for water adsorption. J. Mater. Chem. A 3, 23664-23669 (2015).

50. Guiglion, P., Butchosa, C. \& Zwijnenburg, M. A. Polymer photocatalysts for water splitting: insights from computational modeling. Macromol. Chem. Phys. 217, 344-353 (2016).

51. Guiglion, P., Monti, A. \& Zwijnenburg, M. A. Validating a density functional theory approach for predicting the redox potentials associated with charge carriers and excitons in polymeric photocatalysts. J. Phys. Chem. C 121, 1498-1506 (2017).

52. Bach, U. et al. Solid-state dye-sensitized mesoporous $\mathrm{TiO}_{2}$ solar cells with high photonto-electron conversion efficiencies. Nature 395, 583-585 (1998).

53. Willkomm, J. et al. Dye-sensitised semiconductors modified with molecular catalysts for light-driven $\mathrm{H}_{2}$ production. Chem. Soc. Rev. 45, 9-23 (2016).

54. Abe, R., Sayama, K., Domen, K. \& Arakawa, H. A new type of water splitting system composed of two different $\mathrm{TiO}_{2}$ photocatalysts (anatase, rutile) and a $\mathrm{IO}_{3}{ }^{-} / \mathrm{I}^{-}$shuttle redox mediator. Chem. Phys. Lett. 344, 339-344 (2001).

55. Wang, Q. et al. Scalable water splitting on particulate photocatalyst sheets with a solarto-hydrogen energy conversion efficiency exceeding 1\%. Nat. Mater. 15, 611-615 
(2016).

56. Tada, H., Mitsui, T., Kiyonaga, T., Akita, T. \& Tanaka, K. All-solid-state Z-scheme in $\mathrm{CdS}-\mathrm{Au}-\mathrm{TiO}_{2}$ threecomponent nanojunction system. Nat. Mater. 5, 782-786 (2006).

57. Goto, Y. et al. A particulate photocatalyst water-splitting panel for large-scale solar hydrogen generation. Joule 2, 509-520 (2018).

58. Schröder, M. et al. Hydrogen evolution reaction in a large-scale reactor using a carbon nitride photocatalyst under natural sunlight irradiation. Energy Technol. 3, 1014-1017 (2015).

59. Slater, A. G. \& Cooper, A. I. Function-led design of new porous materials. Science 348, $8075(2015)$

60. Evans, A. et al. Seeded growth of single-crystal two-dimensional covalent organic frameworks. Science 10.1126/science.aar7883 (2018).

61. Teets, T. S. \& Nocera, D. G. Photocatalytic hydrogen production. Chem. Commun. 47, $9268(2011)$.

62. Hisatomi, T., Kubota, J. \& Domen, K. Recent advances in semiconductors for photocatalytic and photoelectrochemical water splitting. Chem. Soc. Rev. 43, 7520-7535 (2014).

63. Weigend, F. \& Ahlrichs, R. Balanced basis sets of split valence, triple zeta valence and quadruple zeta valence quality for $\mathrm{H}$ to $\mathrm{Rn}$ : design and assessment of accuracy. Phys. Chem. Chem. Phys. 7, 3297-3305 (2005).

64. Frisch, M. J. et al. Gaussian 16 Revision A.03. (Gaussian, Inc., 2016)

65. Hirata, S. \& Head-Gordon, M. Time-dependent density functional theory within the Tamm-Dancoff approximation. Chem. Phys. Lett. 314, 291-299 (1999). 
66. Scalmani, G. \& Frisch, M. J. Continuous surface charge polarizable continuum models of solvation. I. General formalism. J. Chem. Phys. 132, 0-15 (2010).

67. Marenich, A. V., Cramer, C. J. \& Truhlar, D. G. Unviersal solvation modle based on solute electron density and a contiuum model of the solvent defind by the bulk dielectric constant and atomic surface tensions. J. Phys. Chem. B. 113, 6378-6396 (2009).

68. Kresse, G. \& Furthmüller, J. Efficient iterative schemes for ab initio total-energy calculations using a plane-wave basis set. Phys. Rev. B 54, 11169-11186 (1996).

69. Perdew, J. P., Burke, K. \& Ernzerhof, M. Generalized gradient approximation made simple. Phys. Rev. Lett. 77, 3865-3868 (1996).

70. Grimme, S., Antony, J., Ehrlich, S. \& Krieg, H. A consistent and accurate ab initio parametrization of density functional dispersion correction (DFT-D) for the 94 elements H-Pu. J. Chem. Phys. 132, 154104 (2010).

71. Grimme, S., Ehrlich, S. \& Goerigk, L. Effect of the damping function in dispersion corrected density functional theory. J. Comp. Chem. 32, 1456 (2011).

72. Heyd, J., Scuseria, G. E. \& Ernzerhof, M. Hybrid functionals based on a screened Coulomb potential. J. Chem. Phys. 118, 8207-8215 (2003).

73. Heyd, J. \& Scuseria, G. E. Efficient hybrid density functional calculations in solids: assessment of the Heyd-Scuseria-Ernzerhof screened Coulomb hybrid functional. $J$. Chem. Phys. 121, 1187-1192 (2004).

74. Heyd, J., Scuseria, G. E. \& Ernzerhof, M. Erratum: "Hybrid functionals based on a screened Coulomb potential". J. Chem. Phys. 124, 219906 (2006).

75. Butler, K. T., Hendon, C. H. \& Walsh, A. Electronic chemical potentials of porous metalorganic frameworks. J. Am. Chem. Soc. 136, 2703-2706 (2014). 
Figure 1. Chemical structures of the organic photocatalysts studied here. a,b, Chemical structures of previously reported photocatalysts (a) $\mathbf{N}_{3}-\mathbf{C O F},{ }^{35}$ (b) $\mathbf{P} 7,{ }^{16}$ and P10. ce, Chemical structures of the COF photocatalysts reported in this work: S-COF, FS-COF and TP-COF.

Figure 2. Crystal structures of FS-COF and S-COF. a,b, Structural models for (a) FS-COF and (b) S-COF with perfectly eclipsed AA stacking patterns, shown parallel to the pore channel along the crystallographic $c$-axis (top) and parallel to the hexagonal layers (bottom). The pores of both COFs are lined with oxygen atoms. c,d, Experimental diffraction patterns (red), profiles calculated from Le Bail fitting (black) and residual (blue) and pattern simulated from structural model (green). Reflection positions are shown by tick marks. Grey, white, blue, red and yellow atoms represent carbon, hydrogen, nitrogen, oxygen and sulfur, respectively.

Figure 3. Evidence for ordered, wettable mesopores in FS-COF. a, Nitrogen adsorption isotherm (filled symbols) and desorption isotherm (open symbols) for FS-COF recorded at $77.3 \mathrm{~K}$. Inset, profile of the calculated pore size distribution for FS-COF. b, TEM image of FSCOF. Hexagonal pore structure with a periodicity of approximately $3.0 \mathrm{~nm}$ outlined by the dashed red box. Scale bars: $50 \mathrm{~nm}$ (inset), and $100 \mathrm{~nm}$ (bottom). c, Water adsorption isotherms (filled symbols) and desorption isotherms (open symbols) for FS-COF, S-COF and TP-COF, measured at $293 \mathrm{~K}$ up to $22.9 \mathrm{mBar}$.

Figure 4. Optical properties, hydrogen evolution rates, and excited state lifetimes for the photocatalysts. a, UV-visible absorption spectra for FS-COF, S-COF, TP-COF and FS-P measured in the solid-state. $\mathbf{b}$, Time-course for photocatalytic $\mathrm{H}_{2}$ production using visible light for FS-COF, S-COF, TP-COF, and FS-P ( $5 \mathrm{mg}$ catalyst in water, $5 \mu \mathrm{L}\left(8 \mathrm{wt} . \% \mathrm{H}_{2} \mathrm{PtCl}_{6}\right), 0.1 \mathrm{M}$ ascorbic acid, $\lambda>420 \mathrm{~nm}$ ). $\mathbf{c}, \mathrm{H}_{2}$ production using visible light for FS-COF over 50 hours total photolysis ( $5 \mathrm{mg}$ catalyst in water, $5 \mu \mathrm{L}$ ( $\left.8 \mathrm{wt} . \% \mathrm{H}_{2} \mathrm{PtCl}_{6}\right), 0.1 \mathrm{M}$ ascorbic acid, $\lambda>420 \mathrm{~nm}$ ). The sample was degassed after 5 and 10 hours to prevent saturation of the detector, then left under continuous illumination for 20 hours and again degassed after 40 and 45 hours. After 35 hours $1.25 \mathrm{mmol}$ of ascorbic acid were added. $\mathbf{d}$, Time-correlated single photon counting experiments for TP-COF, FS-COF and FS-P in water. The samples were excited with a $\lambda_{\text {exc }}=$ $370.5 \mathrm{~nm}$ laser and emission was observed at $\lambda_{\text {em }}=550 \mathrm{~nm}$.

Figure 5. Electronic structure calculations provide insights into the photocatalytic water splitting activities of the COFs. a,b, (TD-)B3LYP predicted IP, EA, IP* and EA* adiabatic potentials (a) of representative fragments of the different COFs (b) in water. c,d, Periodic DFT (HSE06) predicted valence band maximum (VBM; red) and conduction band minimum (CBM; blue) of the COFs with respect to a common vacuum level (c); both eclipsed (AA) and staggered (AB) stacking arrangements, illustrated in (d), were considered. For FS-COF, multiple AA stacked structures were generated, with calculated VBM and CBM for each individual stacking shown as black horizontal lines in (c), for which the assignment is shown in Supplementary Fig. 93. The dashed, coloured lines in $\mathbf{a}$ and $\mathbf{c}$ indicate the potentials for the different solution reactions: green, proton reduction; orange, two-hole $\left(\mathrm{A} / \mathrm{H}_{2} \mathrm{~A}\right)$ and one-hole $\left(\mathrm{HA} \cdot / \mathrm{H}_{2} \mathrm{~A}\right)$ oxidation of ascorbic acid; magenta, overall water oxidation. All solution potentials shown are for $\mathrm{pH} 2.6$, the experimentally measured $\mathrm{pH}$ of a $0.1 \mathrm{M}$ ascorbic acid solution. 
Figure 6. Dye sensitization of FS-COF and hydrogen evolution from an FS-COF film. a, Time-course for photocatalytic $\mathrm{H}_{2}$ production using visible light for FS-COF, a neat, nearinfrared dye (WS5F), and a dye-sensitized COF (FS-COF+WS5F); $5 \mathrm{mg}$ material in water, $5 \mu \mathrm{L}$ (8 wt. $\left.\% \mathrm{H}_{2} \mathrm{PtCl}_{6}\right), 0.1 \mathrm{M}$ ascorbic acid, $\lambda>420 \mathrm{~nm}$ ). b. External quantum efficiencies (EQE) at three different incident light wavelengths for FS-COF and FS-COF+WS5F (5 mg catalyst in water, $5 \mu \mathrm{L}$ ( $8 \mathrm{wt}$. $\left.\% \mathrm{H}_{2} \mathrm{PtCl}_{6}\right), 0.1 \mathrm{M}$ ascorbic acid, $\lambda=420 \pm 10, \lambda=600 \pm 45$ and $700 \pm 10 \mathrm{~nm}$ irradiation; Supplementary Fig. 43). c, Solid-state UV-vis spectra for FS-COF, WS5F and FS-COF+WS5F. d, Relative energy levels as calculated for ascorbic acid, FS-COF, and a near-infrared dye, WS5F; the dashed, green and orange lines indicate the potentials for proton reduction and the two-hole oxidation of ascorbic acid in solution, respectively. e,f, Photocatalytic $\mathrm{H}_{2}$ production using FS-COF films: e, Longer-term hydrogen evolution experiments for a COF film produced with a single drop-cast cycle. f, Photograph showing, from left to right, solid FS-COF and colloidal dispersions in DMF, water, and acetone, respectively (left), see also Supplementary Figs. 33 \& 87; FS-COF film on glass producing hydrogen (right) (20 drop-casting cycles, $0.1 \mathrm{M}$ ascorbic acid, solar simulator AM1.5G, class ABA; see also video file, Supplementary Movie 1).

Table 1. Photophysical properties and hydrogen evolution rates (HERs) for the COF photocatalysts.

\begin{tabular}{|c|c|c|c|c|}
\hline Photocatalyst & $\begin{array}{l}\text { Degree of } \\
\text { crystallinity }\end{array}$ & $\begin{array}{c}\text { Optical } \\
\text { gap }^{[\mathrm{b}]} \\
/ \text { eV }\end{array}$ & $\begin{array}{c}\text { Hydrogen evolution } \\
\text { rate }^{[\mathrm{cc}]} \\
/ \mathbf{m m o l ~ g}^{-1} \mathbf{h}^{-1}\end{array}$ & $\begin{array}{c}\text { Hydrogen } \\
\text { evolution rate } \\
\text { relative to } \\
\text { FS-COF }\end{array}$ \\
\hline TP-COF ${ }^{[\mathrm{a}]}$ & Crystalline & 2.28 & $1.60 \pm(0.08)$ & $16 \%$ \\
\hline $\mathrm{S}-\mathrm{COF}^{[\mathrm{a}]}$ & Crystalline & 2.10 & $4.44 \pm(0.14)$ & $43 \%$ \\
\hline FS-COF ${ }^{[a]}$ & Crystalline & 1.85 & $10.1 \pm(0.3)$ & - \\
\hline FS-P[a] & Amorphous & 1.88 & $1.12 \pm(0.16)$ & $11 \%$ \\
\hline $\mathrm{N}_{3}-\mathrm{COF}^{[35]}$ & Crystalline & 2.60 & $0.47 \pm(0.06)$ & $4.6 \%$ \\
\hline $\mathrm{P} 7^{[16]}$ & Semi-crystalline & 2.70 & $0.84 \pm(0.06)^{[\mathrm{d}]}$ & $8.3 \%$ \\
\hline $\mathrm{P} 10$ & Semi-crystalline & 2.55 & $1.48 \pm(0.1)^{[\mathrm{d}]}$ & $15 \%$ \\
\hline FS-COF + WS5F $^{[a]}$ & Crystalline & [e] & $16.3 \pm(0.29)$ & $161 \%$ \\
\hline $\mathrm{FS}-\mathrm{COF}+\operatorname{Eosin} \mathrm{Y}^{[\mathrm{a}]}$ & Crystalline & [e] & $16.1 \pm(0.34)$ & $159 \%$ \\
\hline FS-P + WS5F $F^{[a]}$ & Amorphous & {$[\mathrm{e}]$} & $0.23 \pm(0.03)$ & $2.3 \%$ \\
\hline FS-P + Eosin $Y^{[a]}$ & Amorphous & [e] & $0.58 \pm(0.08)$ & $5.8 \%$ \\
\hline
\end{tabular}

[a] This work; ${ }^{[b]}$ Calculated from the on-set of the solid absorption spectrum; ${ }^{[c]}$ All rates measured using the same instruments, optical set-up and reaction conditions: $5 \mathrm{mg}$ COF catalyst, $5 \mu \mathrm{L}$ ( $8 \mathrm{wt}$. \% $\left.\mathrm{H}_{2} \mathrm{PtCl}_{6}\right), 25 \mathrm{~mL}$ ascorbic acid aqueous solution $(0.1 \mathrm{M}), 300 \mathrm{~W}$ Xe light source equipped with $\lambda>420 \mathrm{~nm}$ cut-off filter. Hydrogen evolution rates (HER) based on average over 5 hours irradiation and normalized to the COF mass; ${ }^{[\mathrm{d}]}$ As for [c], but with no additional platinum catalyst added (Supplementary Fig. 35); HER for P10 in the presence of Pt was $1.92 \mathrm{mmol} \mathrm{g}^{-1} \mathrm{~h}^{-1}$. ${ }^{[\mathrm{e}]}$ Effective optical gap was not measurable due to the intense absorption of the organic dye. 
a

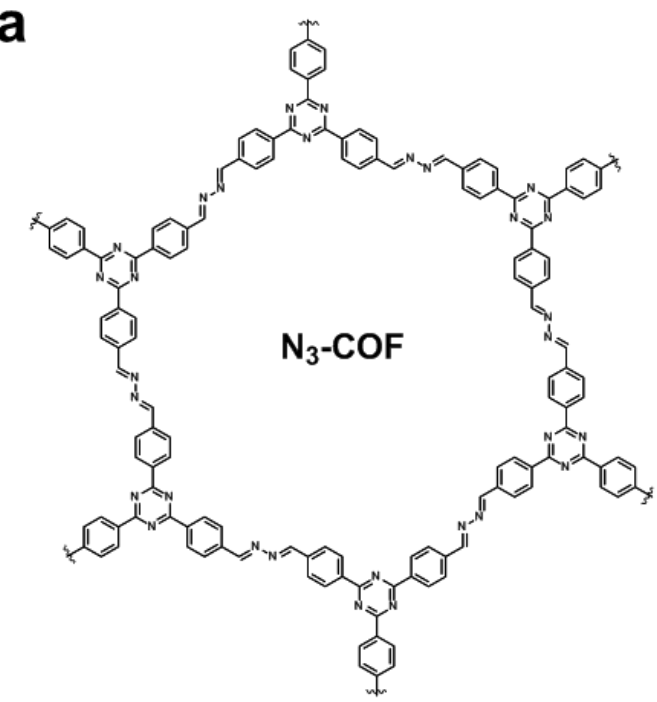

b

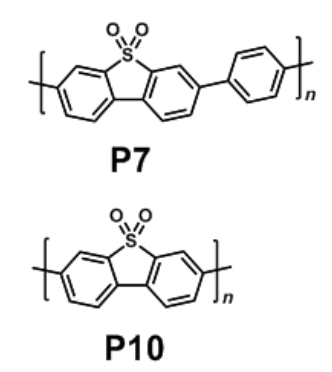

C
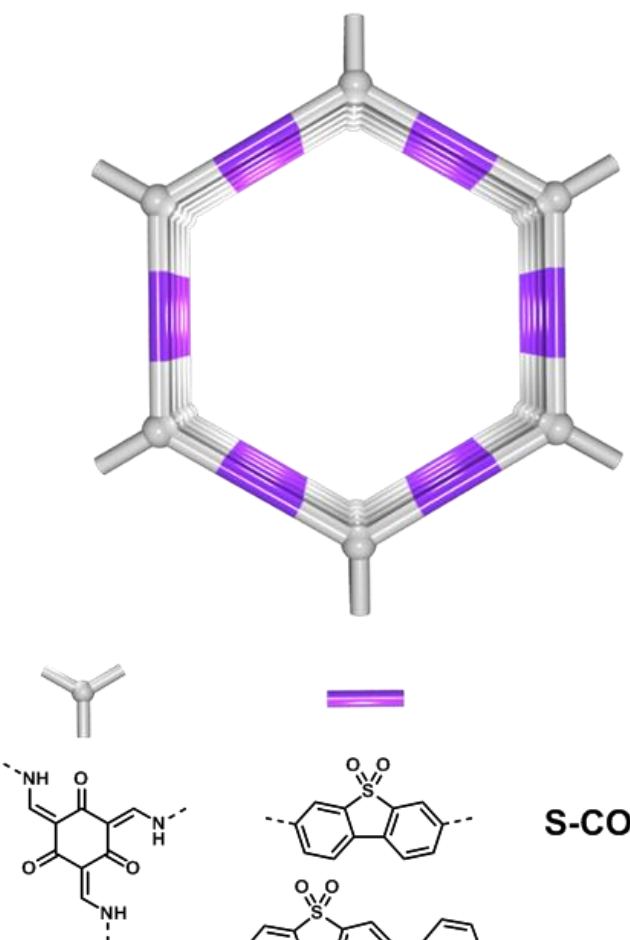

$\overline{=}$

S-CoF

FS-COF

-. 


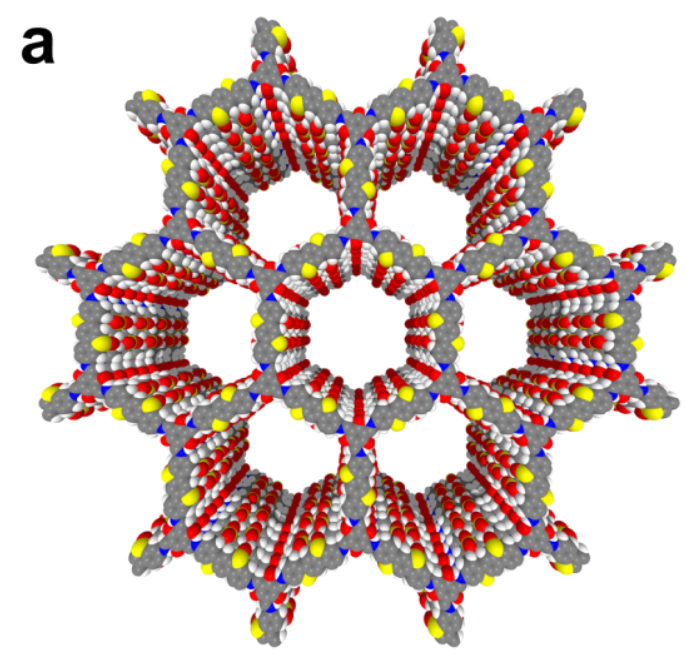

b
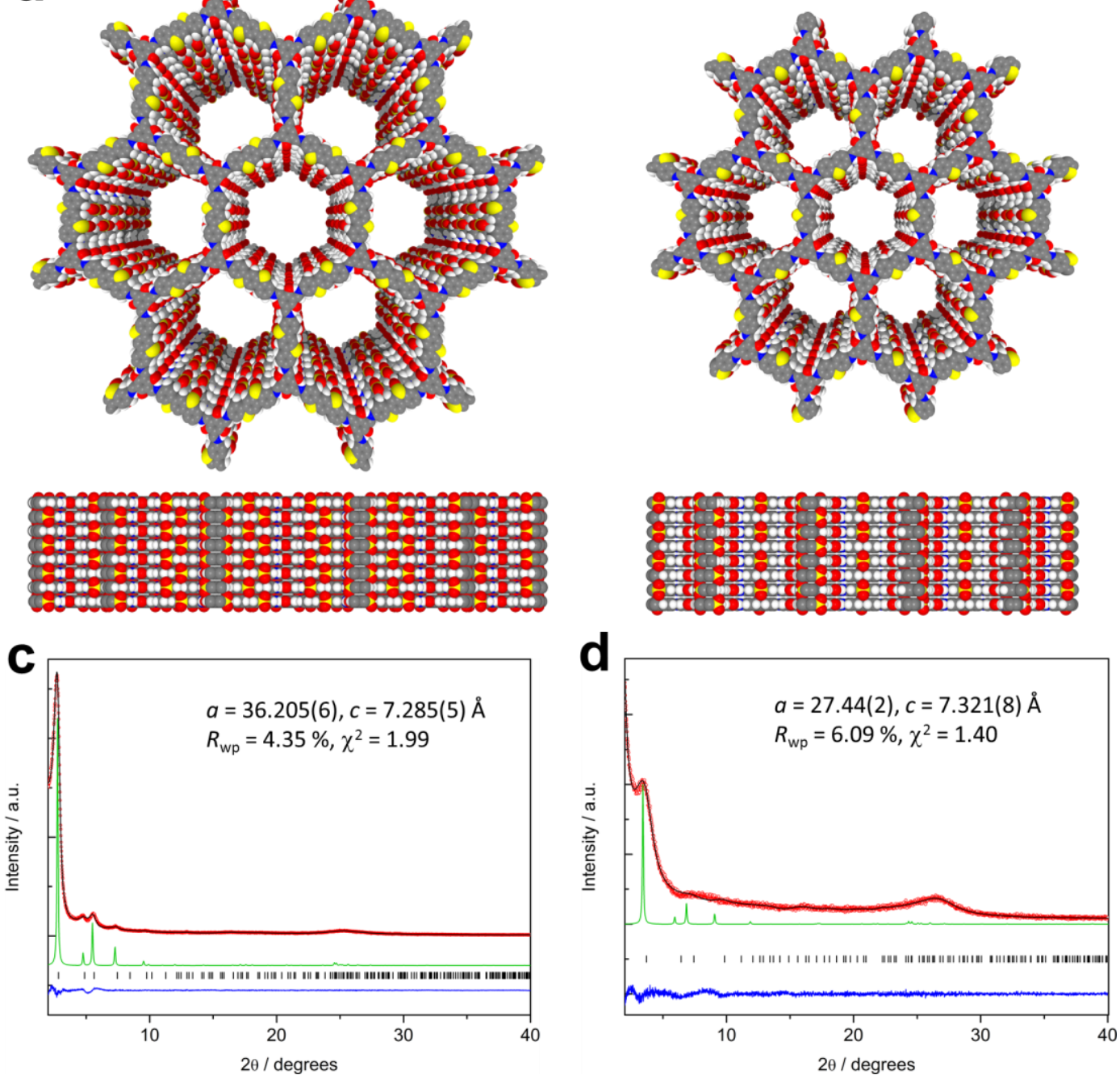

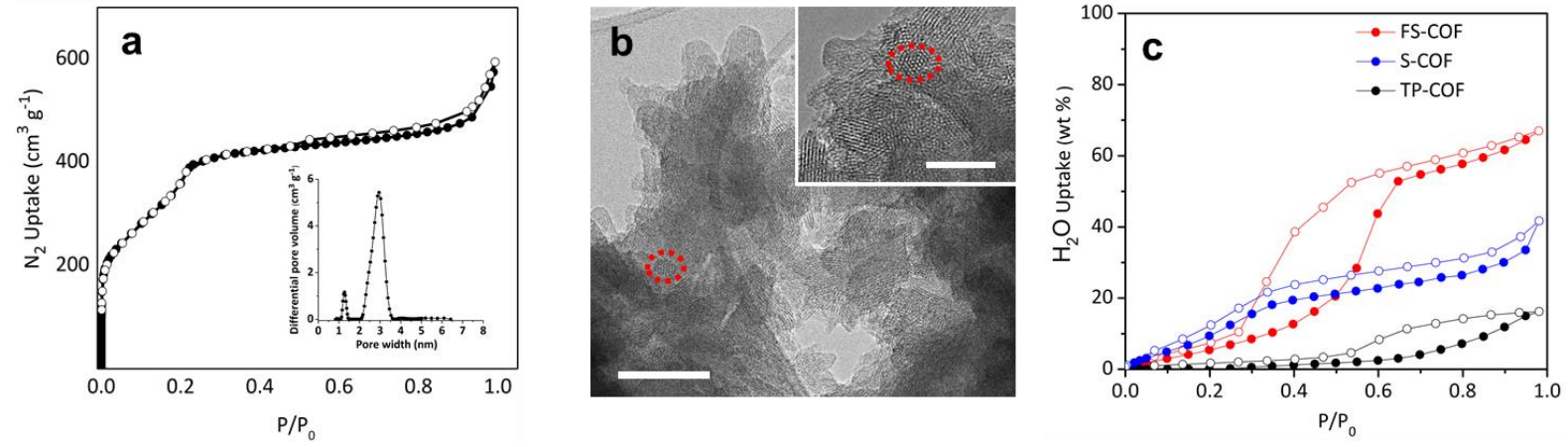

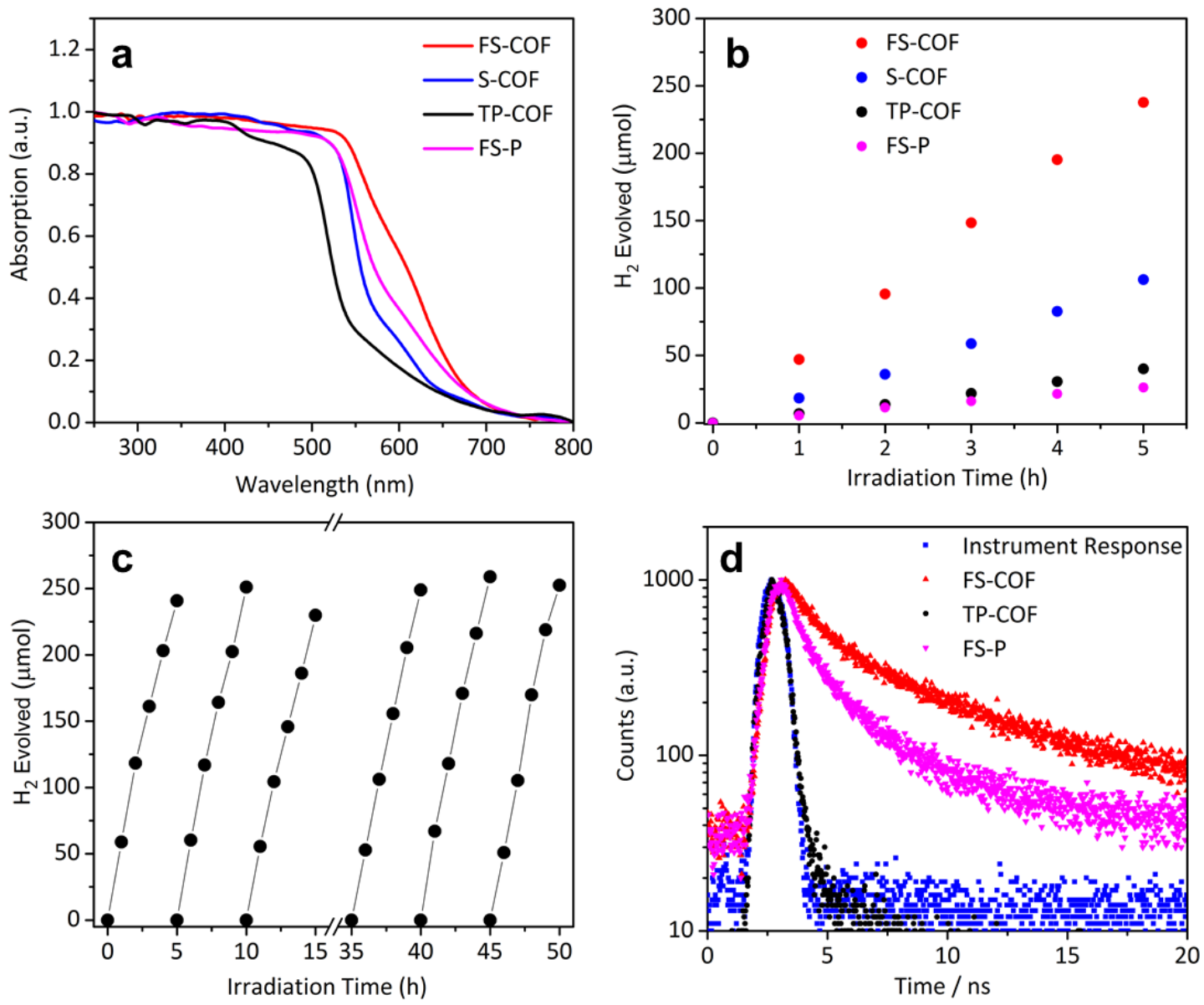

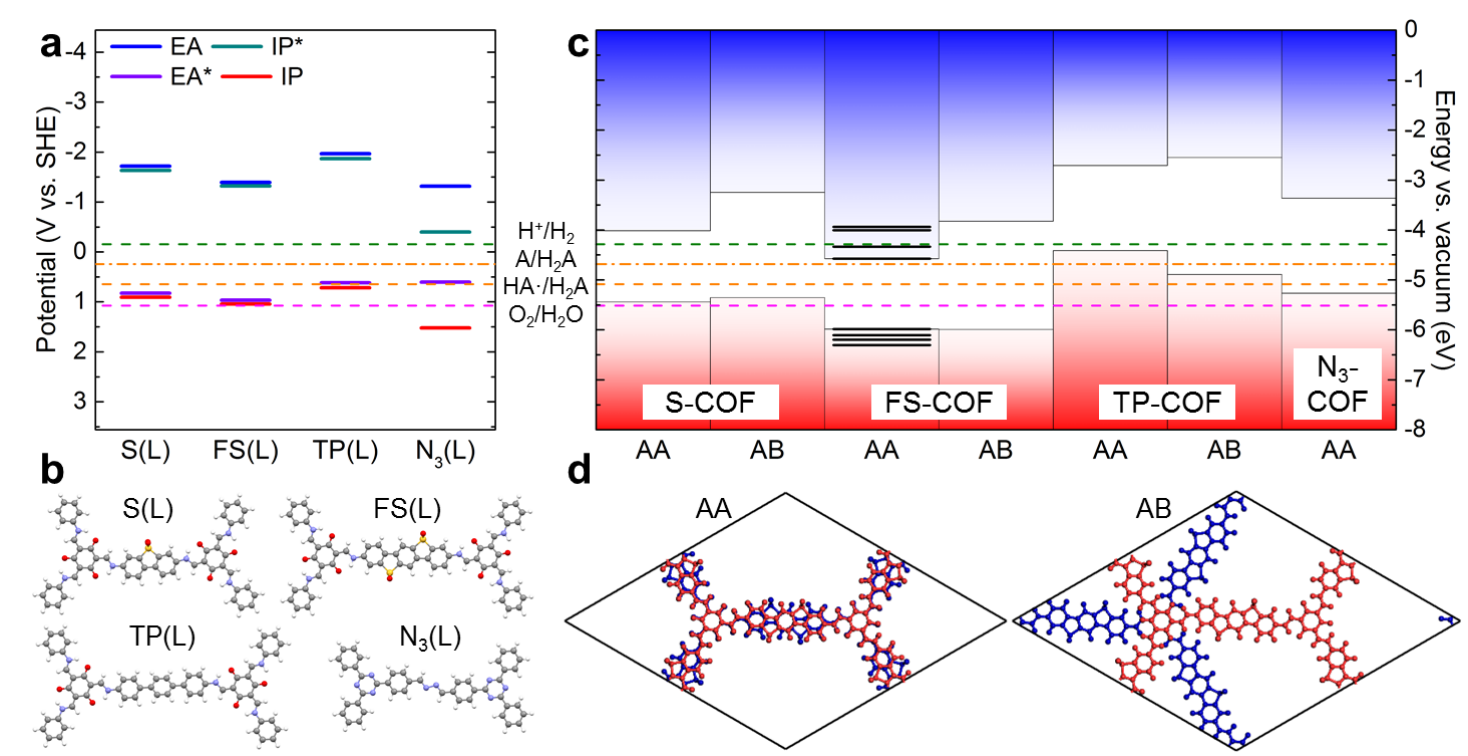

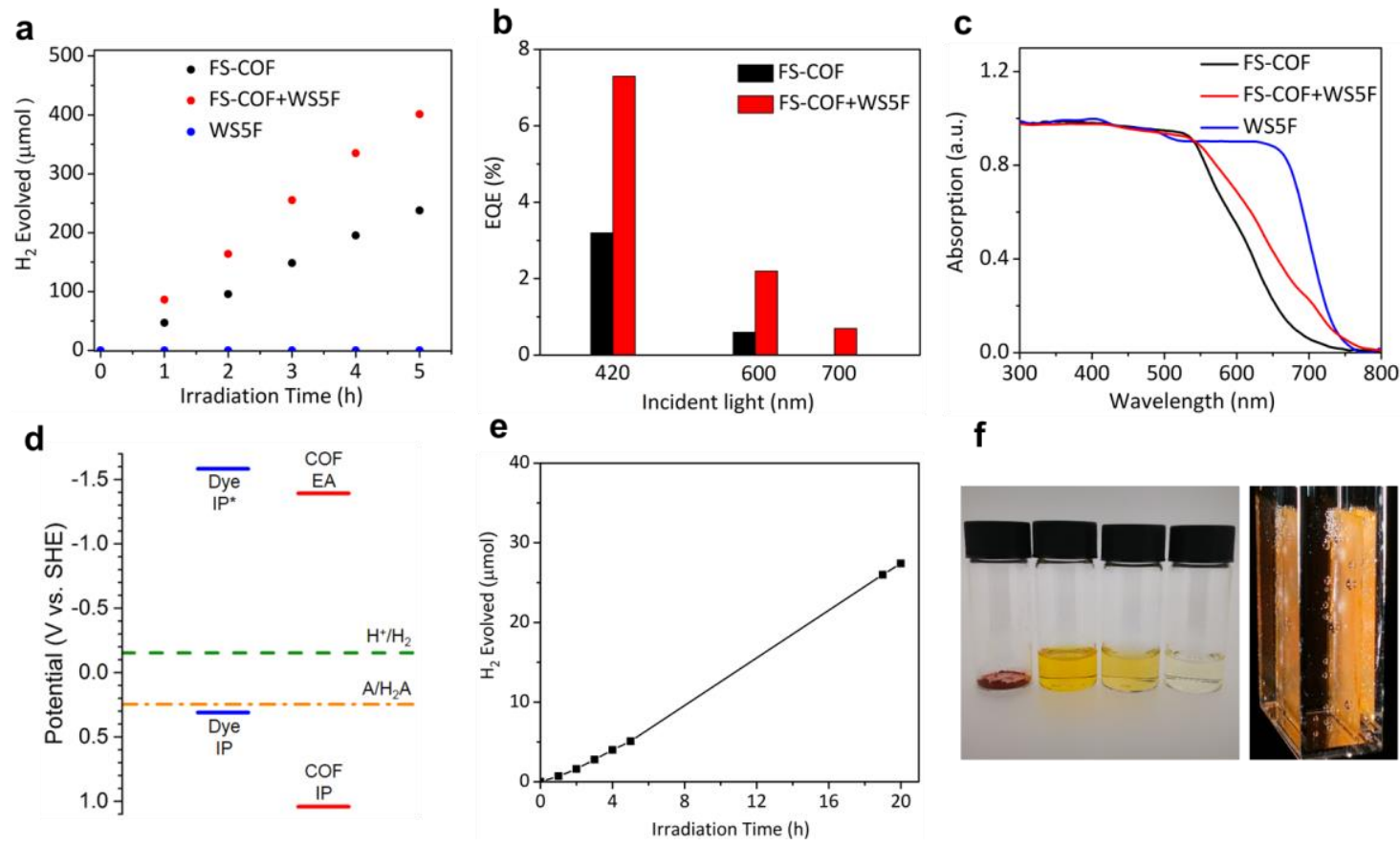

f

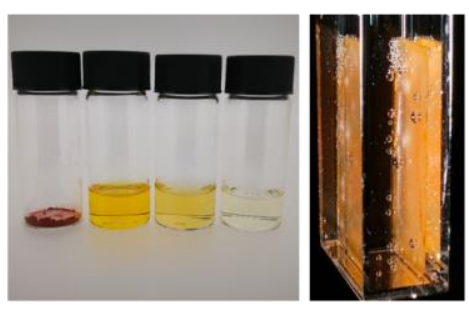




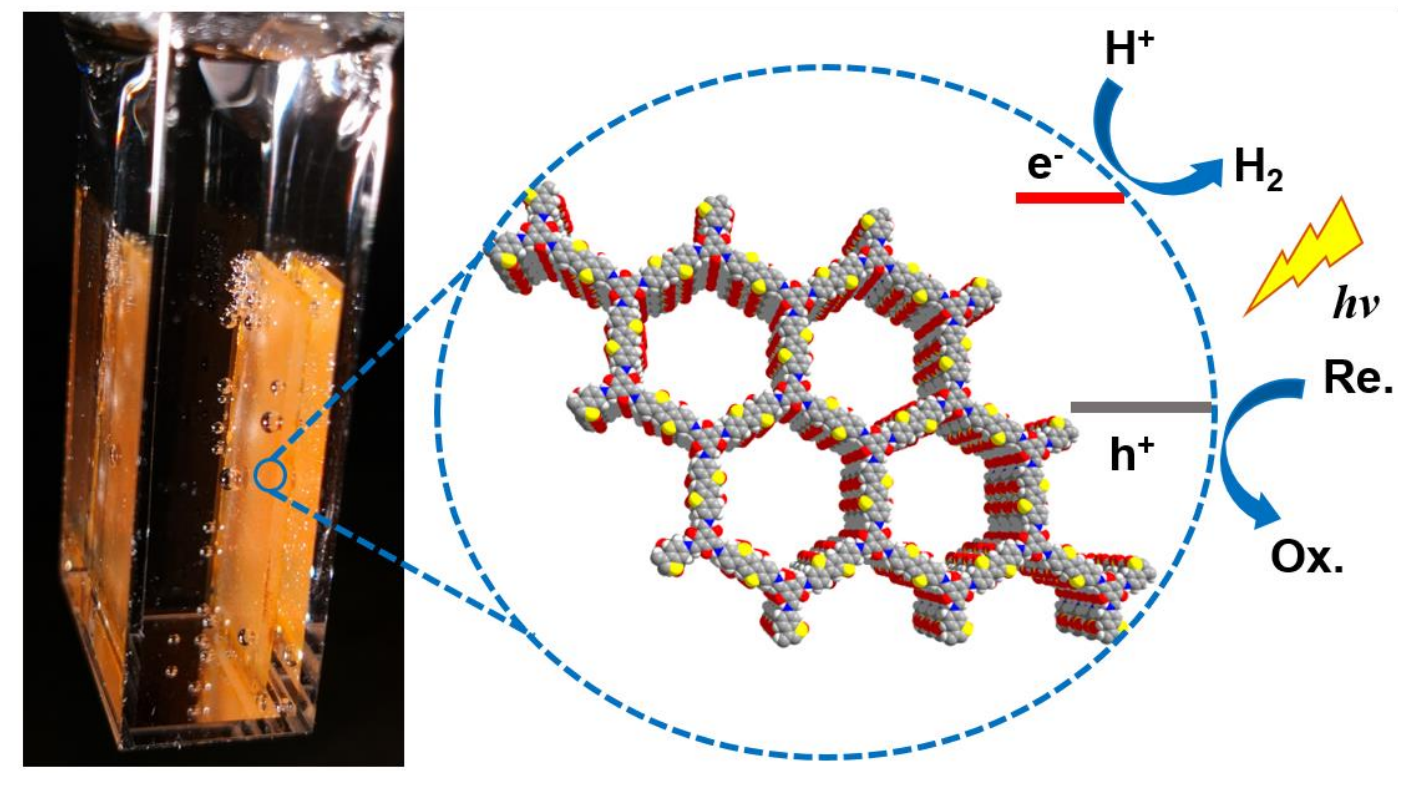

